\title{
Reversible data hiding using least square predictor via the LASSO
}

Hee Joon Hwang ${ }^{1}$, SungHwan Kim² and Hyoung Joong Kim"

\begin{abstract}
Reversible watermarking is a kind of digital watermarking which is able to recover the original image exactly as well as extracting hidden message. Many algorithms have aimed at lower image distortion in higher embedding capacity. In the reversible data hiding, the role of efficient predictors is crucial. Recently, adaptive predictors using least square approach have been proposed to overcome the limitation of the fixed predictors. This paper proposes a novel reversible data hiding algorithm using least square predictor via least absolute shrinkage and selection operator (LASSO). This predictor is dynamic in nature rather than fixed. Experimental results show that the proposed method outperforms the previous methods including some algorithms which are based on the least square predictors.
\end{abstract}

\section{Introduction}

Reversible data hiding technique embeds data into host signal such as text, image, audio, or video with the functionality of recovering original signal as well as extracting hidden data. It can be utilized for various purposes such as military or medical image processing which requires the integrity of the original image.

Difference expansion invented by Tian [1] is a fundamental technique for reversible data hiding that expands the difference value of a pair of pixels to hide one bit per pair. Alattar [2] proposed an embedding method using difference values among a triplet of pixels to hide two bits per triplet. In addition, he showed that three bits can be hidden into a quad [3].

After that, prediction error expansion (PEE) was proposed by Thodi and Rodriguez [4] as a generalized form of difference expansion. Prediction error which means difference between the original pixel and the predicted pixel is expanded for reversible data hiding. Probability distribution function of the prediction errors is sharper and narrower than that of the simple difference of the pixel values, which is better for reversible data hiding. Small distortion with large embedding capacity is a desirable feature of the reversible data hiding. Thodi and Rodriguez [4] also used the median edge detector

\footnotetext{
*Correspondence: khj-@korea.ac.kr

${ }^{1}$ Graduate School of Information Security, Korea University, Seoul, South

Korea

Full list of author information is available at the end of the article
}

(MED) as a predictor introduced for the lossless image compression standard such as JPEG-LS [5].

Chen et al. [6] compared the performances of many predictors such as MED, 4th-order gradient-adjusted predictor (GAP) employed in context-based adaptive lossless image compression (CALIC) [7], and the full context prediction [6] using the average of the four closest neighbored pixels. Full context prediction using rhombus pattern and sorting method is also proposed in [8] by Sachnev et al. These are all classified as fixed predictor in [9].

Full context rhombus predictor has the best performance among all fixed predictors [6]. That is the reason why many papers implemented embedding algorithm based on the full context rhombus predictor [10-13].

On the other hand, various papers [14-16] focused on improving PSNR performance in small embedding capacity. Dragoi and Coltuc [16] utilized the rhombus pattern even in small embedding capacity and obtain a good result. However, the problem of these methods have small embedding capacity. The optimization scheme such as least square approach is essential for high embedding capacity as well as small image distortion.

Adaptive predictors using least square approach are also introduced in many papers $[17,18]$ and applied in reversible data hiding $[19,20]$. Edge-directed prediction (EDP) is a least square predictor which optimizes the prediction coefficients locally inside a training set. Kau and Lin [17] proposed edge-look-ahead (ELA) scheme 
using least square prediction with efficient edge detector to maximize the edge-directed characteristics. Wu et al. improved the least square predictor by determining the order of predictor and support pixels adaptively [18].

All of these predictors' performance was properly compared in several papers [17, 21]. However, all these adaptive predictors' performance was not able to outperform the simple rhombus predictor, because those had to use the only previous pixels of the target pixel while the rhombus predictor utilized four neighboring pixels [6].

Dragoi and Dinu [9] and Lee et al. [20] improved the least square predictor by modifying traditional training set consisting of only previous pixels of the target pixel. Dragoi and Dinu utilized training set with pixels in square shaped block surrounding the target pixel. Only half of the pixels within the block are original pixels and the other half are modified ones after data embedding. Least square predictor in [20] includes four neighboring pixels as well as a subset of previous pixels for the training set. Their predictor divides an image into cross and dot sets. When embedding data in the cross set, predictor uses training set consisting of the original pixels, while in the dot set, it uses half-modified training set. Therefore, both techniques clearly outperform the previous least square predictor [19] and the rhombus patterned fixed predictor [8].

Least square approach is one of the most advanced types of adaptive predictor in reversible data hiding. However, in statistics, it is well known that penalized regression approach which accompanies efficient variable selection can lead to finding smaller and more necessary supports for the purpose of good prediction accuracy.

In this paper, we propose a reversible data hiding technique using the least square predictor via penalized regression method called the least absolute shrinkage and selection operator (LASSO) to overcome weaknesses of the existing prediction methods.

In addition to the difference expansion method, histogram shifting (HS) method [22] has played important role in the reversible data hiding community. It provides less distortion to that difference expansion method. However, in most cases, two methods are used as a single algorithm. One of the mainstreams of the reversible data hiding is utilizing a combination of histogram shifting and prediction error expansion (PEE $+\mathrm{HS}$ ) with good predictors. Comprehensive explanation of the various algorithms and their application can be available at [23].

The organization of this paper is as follows: section 2 explains the related works on which the proposed method is based on, section 3 presents the proposed algorithm, section 4 presents experimental results to show that the proposed algorithm is superior to other methods, and section 5 presents the conclusion.

\section{Related works}

2.1 Two-stage embedding scheme using rhombus pattern Among fixed predictors, rhombus scheme has the best performance compared to other kinds of predictors such as GAP, MED, and CALIC [6]. In the rhombus pattern, two-stage embedding scheme was proposed by Sachnev et al. [8]. They divided an image into two kinds of nonoverlapping sets of pixels with a rhombus pattern. In other words, it consists of two sets, so-called cross set and dot set as shown in Fig. 1. Their predicted value is obtained by the average value of four neighboring pixels around the target pixel. For example, a pixel which belongs to the cross set is predicted by the four closest pixels which belong to the dot set as shown in Fig. 1. Because of its effective grouping, it succeeds in

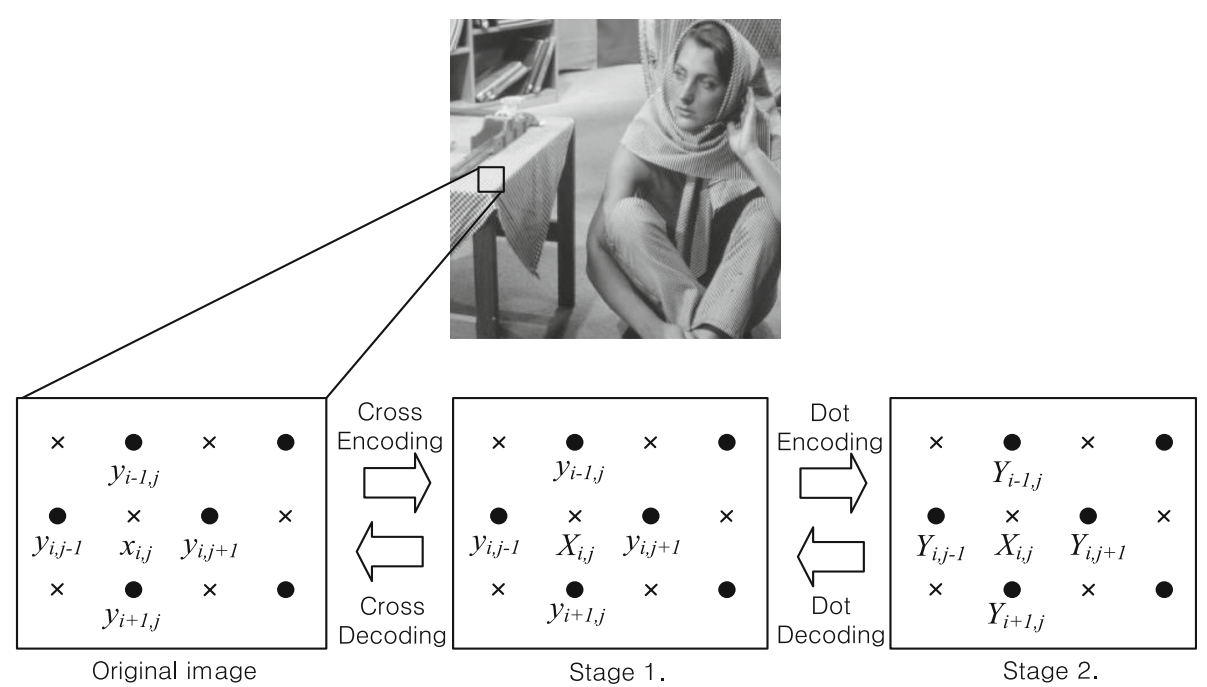

Fig. 1 Two-stage embedding scheme 
forming full context prediction and makes the outstanding improvement of the prediction accuracy over all other prediction methods which have support pixels including only the previous region of the target pixel. In other words, it is very difficult to design more advanced rhombus pattern predictor, despite the highorder modeling such as CALIC [7] and piecewise 2D auto-regression (P2AR) methods having many support pixels $[6,17,18]$. It turns out that accuracy of the predictor depends on not only the order but also the location of support pixels. Therefore, we adopt the rhombus pattern predictor and the two-stage embedding scheme in this paper.

\subsection{Linear prediction}

\subsubsection{Least square approach}

The coefficients for support pixels are computed adaptively by the least square (LS) methods in linear prediction. It is one of the most advanced types of adaptive predictor, and it normally can provide better performance than fixed predictors $[6,9]$. The fixed predictor uses the fixed coefficients. However, adaptive predictor computes the coefficients dynamically according to the context.

In this paper, we use $x(n)$ to denote a current target pixel, where $n$ is the spatial coordinate in an image. Suppose that an image is scanned in a raster-scanning order, and $x(n)$ is predicted by its causal neighboring pixels. According to the $N$-th order Markovian property, the predicted value is computed by $N$ neighboring pixels as follows:

$$
x_{p}(n)=\sum_{k=1}^{N} \beta(k) x(n-k)
$$

where $\beta(k)$ is a prediction coefficient for the $k$-th support pixel.

LS predictor works adaptively to the local features around the target pixel on a pixel-by-pixel basis [17]. In other words, the relations between each training set pixel and its support pixels are exploited usefully for predicting the relation between the target pixel and its support pixels as shown in Fig. 2.

The prediction coefficients are optimized locally inside each training set. A convenient choice of the training set is shown in Fig. 2 enclosed by blue lines, which contains $M=2 L(L+1)$ causal neighboring pixels. Let us denote the pixels in the training set by a $M \times 1$ column vector as follows:

$$
Y=\left[\begin{array}{llll}
y_{1}(n) & y_{2}(n) & \cdots & y_{M}(n)
\end{array}\right]^{T}
$$

Each pixel in the training set has the support pixels which consist of the $N$ closest red-colored cross pixels as shown in Fig. 2. Then, the pixels in the training set and their support pixels would form an $M \times N$ matrix $X$ as follows:

$$
X=\left[\begin{array}{ccc}
x_{1}(n-1) & \cdots & x_{1}(n-N) \\
\vdots & \cdots & \vdots \\
x_{M}(n-1) & \cdots & x_{M}(n-N)
\end{array}\right]
$$

where $x_{j}(n-k)$ is the $k$-th support pixel of training set pixel $x_{j}(n)$. The prediction coefficients are obtained through LS optimization inside the training set:

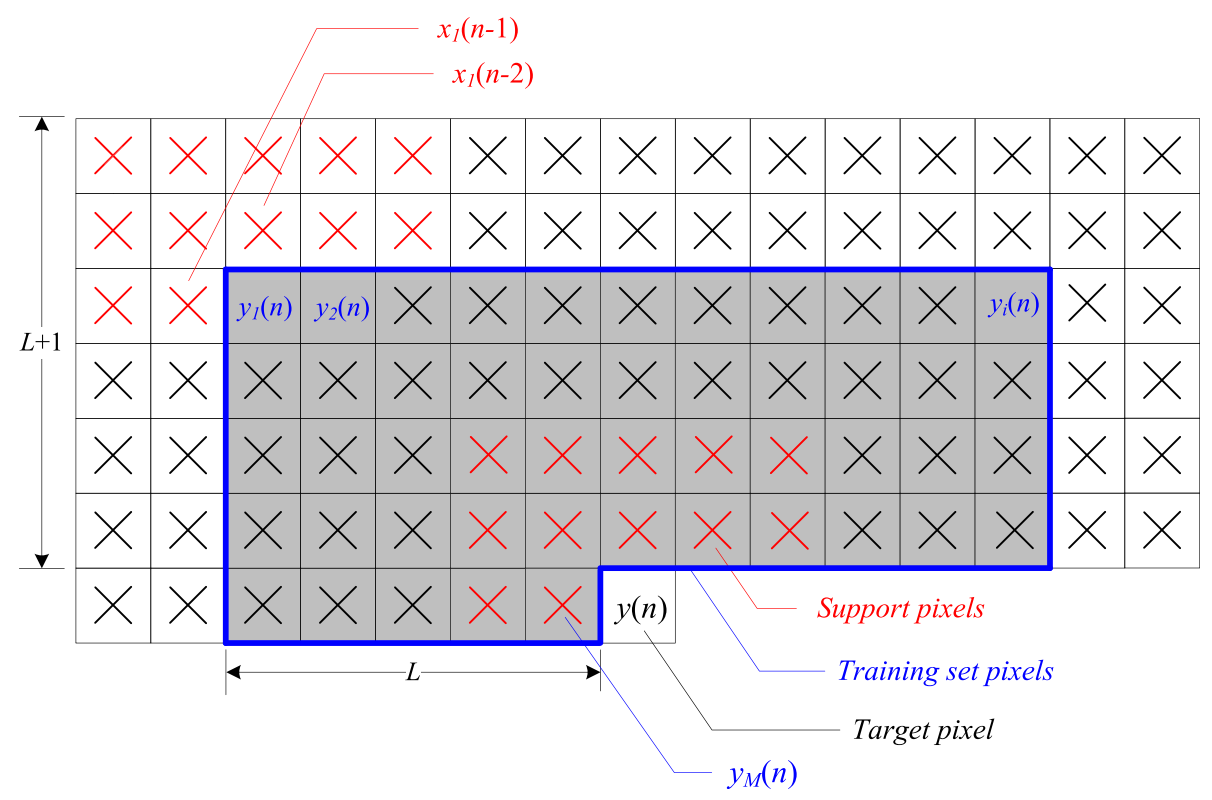

Fig. 2 An example of a training set (in gray color) and a set of support pixels (with red crosses) 


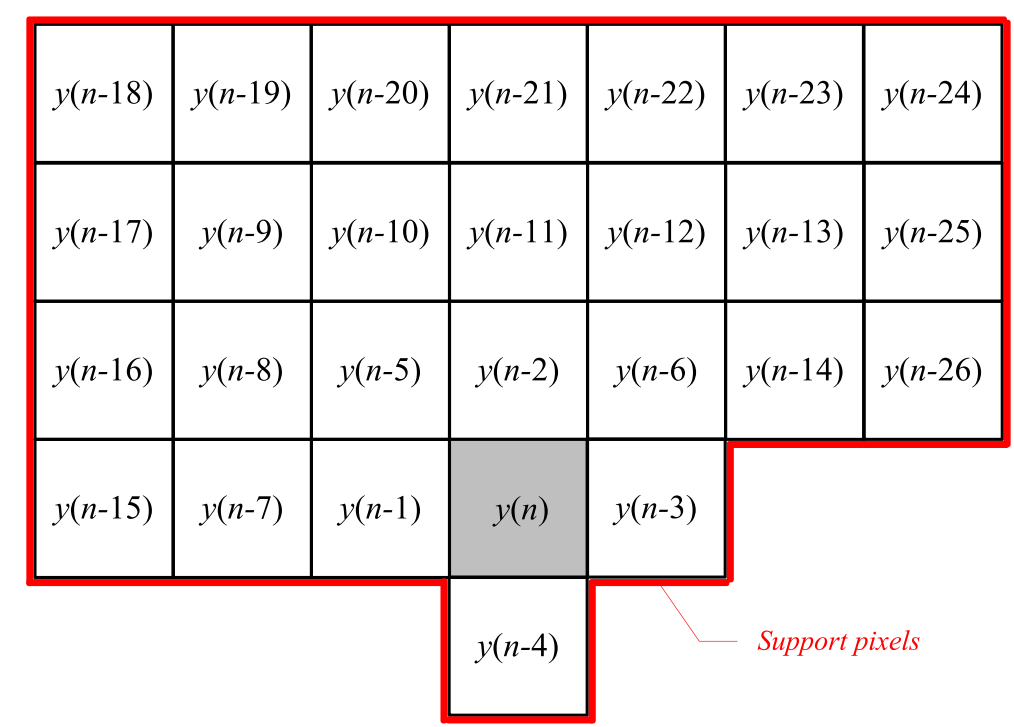

Fig. 3 A set of support pixels surrounding a target pixel (in the red box) of the proposed predictor $(N=26)$

$$
\min \|Y-X \beta\|
$$

It is a well-known fact that the P2AR optimization has a closed-form solution as follows:

$$
\beta=\left(X^{T} X\right)^{-1}\left(X^{T} Y\right)
$$

where $\beta=\left[\begin{array}{llll}\beta(1) & \beta(2) & \cdots & \beta(N)\end{array}\right]^{T}$ is the optimized prediction coefficients which have to be multiplied by the support pixels. It provides an optimal prediction locally inside the training set.

\subsubsection{Penalized regression using LASSO}

Penalized regression methods aim at simultaneous variable selection in coefficient estimation. In practice, even if the sample size is small, a large number of support pixels are typically included to mitigate modeling biases. With such a large number of support pixels, there might exist multicollinearity problems among explanatory variables $X$. Thus, selecting an appropriate size of the support pixels in a subset is desirable. Penalized regression can be an effective tool for such a selection.

Among methods that do both continuous shrinkage and variable selection, a promising technique called the LASSO was proposed by Tibshirani [24]. The LASSO is a penalized least squares procedure that minimizes the residual sum of squares (RSS) subject to the nondifferentiable constraint expressed in terms of the $L_{1}$ norm of the coefficients. That is, the LASSO estimator is given by

$$
\beta_{L}=\arg \min (Y-X \beta)^{\prime}(Y-X \beta)+\lambda \sum_{j=1}^{p}\left|\beta_{j}\right|
$$

where $\lambda \geq 0$ is a tuning parameter.
Regarding the choice of the best parameter $\lambda$ in Eq. (5), we utilize the Bayesian information criteria (BIC) introduced by Schwarz [25], through which we select a model that maximizes the posterior probability $P[$ model $Y]$, where the model is subject to $\lambda$. Schwarz presents the approximation to the posterior probability for the iid (independent and identically distributed) case:

$$
B I C=-2 \cdot l(\hat{\beta})+p \cdot \log (n),
$$

where $l(\hat{\beta})$ is the log-likelihood equation evaluated at the maximum likelihood estimate under the model of interest, $p$ is the degree of freedom of our estimator, and $n$ is the number of observations. It is interesting to note that the BIC adjusts the trade-off between the likelihood and the degree of sparseness so that the model maximizes the posterior probability.

\section{Proposed algorithm}

Compared to existing reversible data hiding methods, the proposed method provides improved performance by using a more accurate predictor. The proposed idea is mainly based on two-stage embedding system of Sachnev et al.'s idea. We focus on improving prediction accuracy by:

- Applying least square predictor which is able to obtain adaptive weigh for each support pixel.

- Applying LASSO penalized regression to least square predictor on purpose of selecting the number and location of support pixels adaptively.

\subsection{Least square predictor based on rhombus scheme} Basically the proposed predictor is based on the twostage embedding scheme using rhombus pattern [8]. It is 


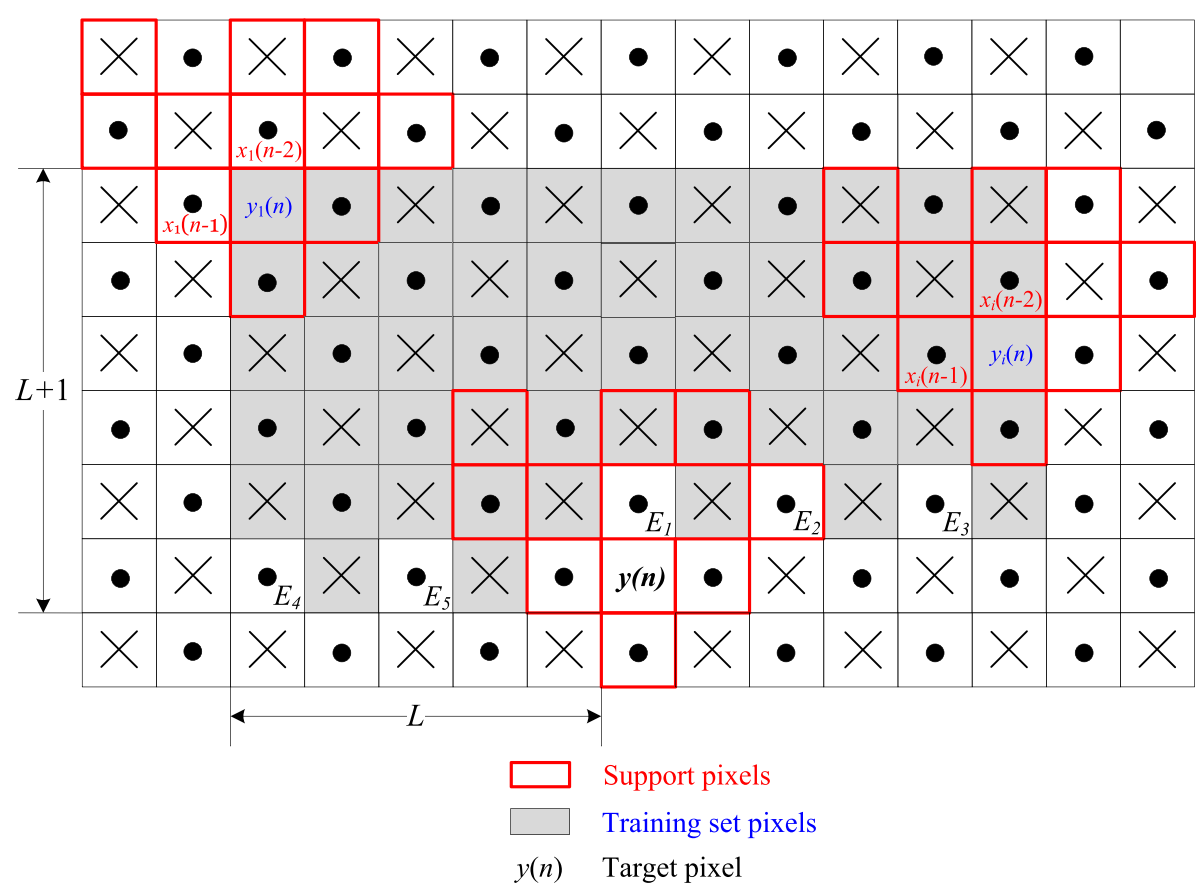

Fig. 4 An example of a training set (in gray color) for prediction $(L=5)$

combined with the LS-based adaptive predictor [17] in the proposed method. Thus, support pixels of the proposed method consist of surrounding pixels around $y(n)$ as shown in Fig. 3. A shape of a training set is shown in Fig. 4 in gray color.

Due to the property of two-stage embedding scheme [8], there are some pixels which should be excluded from the training set. Suppose that we embed a bit in dot set first. In Fig. 4, (in case of $N=9$ ), basically all pixels of the cross set in the past of target pixel can be

\begin{tabular}{|c|c|c|c|c|}
\hline 113 & 46 & 84 & 59 & 73 \\
\hline 146 & 95 & 84 & 141 & 101 \\
\hline 98 & 124 & 83 & 123 & \\
\hline
\end{tabular}

included in the training set and all pixels in the dot set such as $E_{1}, E_{2}, E_{3}, E_{4}, E_{5}$, and $E_{6}$ should be excluded from the training set because those pixels break reversibility. In other words, those pixels use at least one support pixel which is located in or behind target pixel.

Table 1 The sample of prediction coefficients for support pixels

\begin{tabular}{llll}
\hline Index $i$ & $x(n-i)$ & \multicolumn{2}{l}{$\beta_{i}$} \\
\cline { 3 - 4 } & & LS-based & LASSO \\
\hline 0 & $\mathrm{~N} / \mathrm{A}$ & -26.393 & -13.395 \\
1 & 124 & -0.022 & 0 \\
2 & 84 & 0.337 & 0.228 \\
3 & 123 & 0.499 & 0.458 \\
4 & 77 & 0.417 & 0.374 \\
5 & 95 & 0.459 & 0.434 \\
6 & 141 & -0.449 & -0.312 \\
7 & 98 & 0.073 & 0 \\
8 & 146 & -0.215 & -0.084 \\
9 & 113 & 0.168 & 0.021 \\
10 & 46 & -0.230 & -0.086 \\
11 & 84 & -0.053 & -0.069 \\
12 & 59 & 0.154 & 0.094 \\
13 & 73 & -0.042 & 0 \\
14 & 101 & 0.153 & 0.072 \\
\hline
\end{tabular}



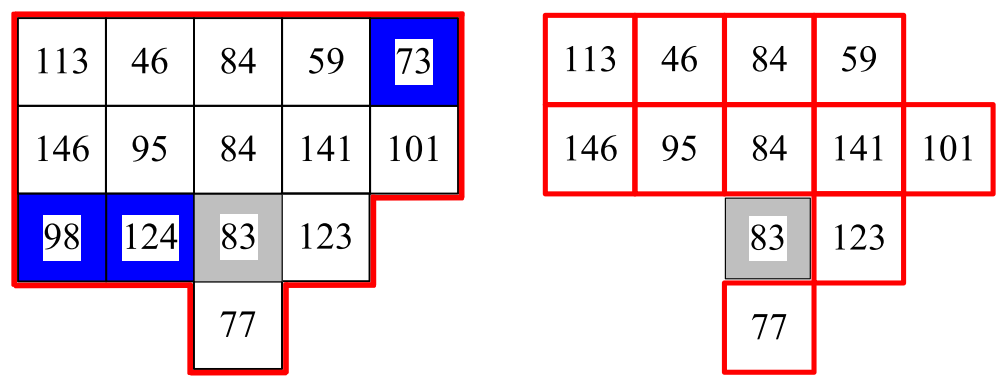

Fig. 6 The result of LASSO penalization: before (left) and after (right)

Suppose that we have $M$ training set pixels excluding the above improper pixels according to the size $T$. Each pixel has $N$ support pixels. Then, it forms an $M \times N$ matrix $X$ as shown in Eq. (3). However, the proposed method applies one more idea to use more proper support pixels for the purpose of improving the accuracy of the LS-based predictor.

\subsection{Applying penalized regression via LASSO}

LS-based prediction method, an adaptive predictor, can be improved by using penalized regression. In the proposed method, LASSO is utilized for penalized regression. LS predictor provides an adaptive coefficient value, but penalized regression can make LS method be more adaptive. By the proposed method, we can penalize and remove some support pixels which are not influential to the target pixel. In other words, we can estimate the location of the most critically influential support pixels as well as their prediction coefficients.

The following example explains how the proposed method achieves better performance. For example, in Fig. 5, when the target pixel value $y(n)$ is 83 , its support pixel values are $x(n-1)=124, x(n-2)=84$, and $x(n-14)$ $=101$ (see Fig. 3). Prediction coefficients are obtained from the LS-based approach and LASSO penalization. After the LASSO penalization, prediction coefficients for the blue colored pixels such as $x(n-1), x(n-7)$, and $x(n-13)$ become zero as shown in Table 1 , which means that the number of the support pixels for the target pixel is reduced as shown in Fig. 6. The left-hand side and the right-hand side show the support pixels before and after the LASSO penalization, respectively. By removing the uncorrelated support pixels, prediction error gets smaller.

Table 1 shows that the coefficients of 124,98 , and 73 are smaller than others in magnitude. Thus,

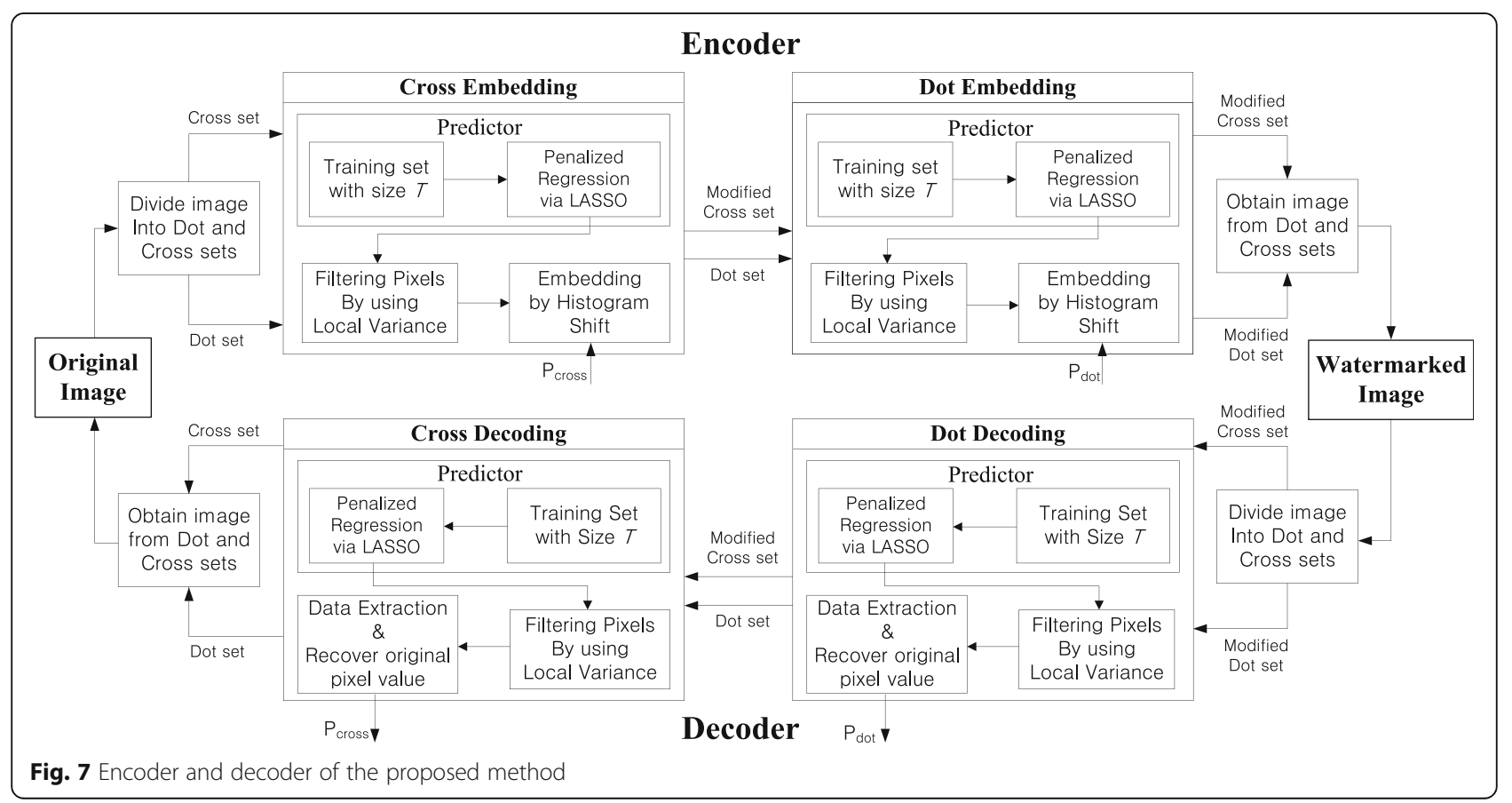




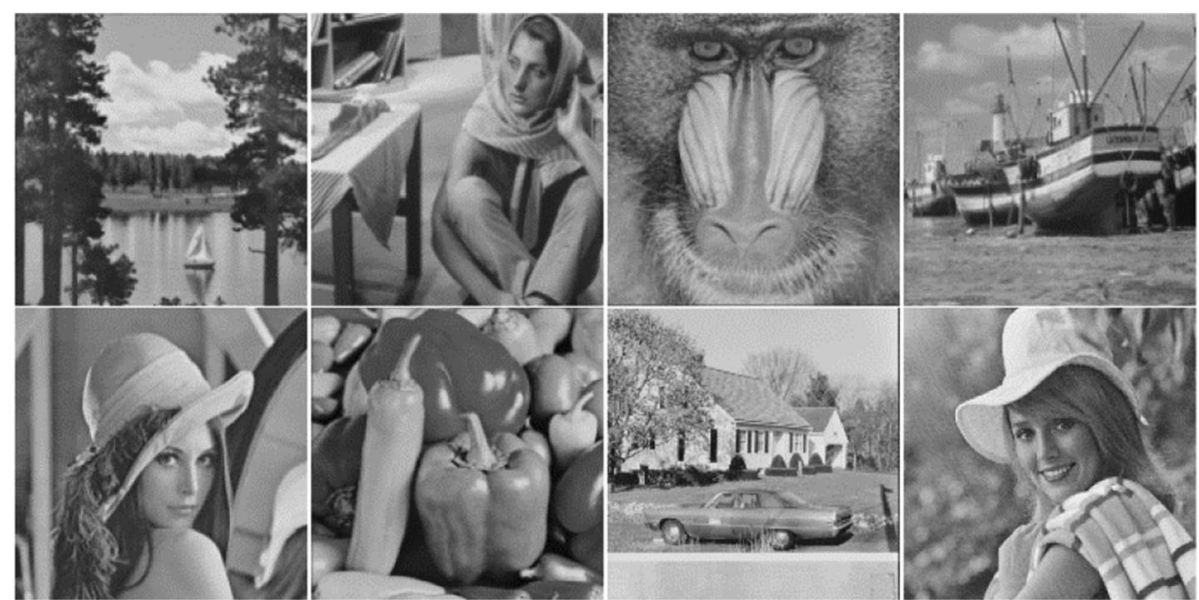

Fig. 8 Standard test images: Sailboat, Barbara, Baboon, Boat, Pepper, Lena, House, Elaine

LASSO assigns 0 values to them and remove those pixels from the support pixels.

The LS-based approach calculates the predicted value $x_{p}$ as 74 , and the LASSO penalization calculates it as 81 according to Eq. (1). LASSO estimates the target pixel more exactly because its given value is 83 .

\subsection{Encoder and decoder}

This section describes the main step of the encoding and decoding processes. The proposed idea is explained more explicitly step by step with the description of the full process.

Table 2 Comparison in terms of average PSNR(dB) for low embedding capacities(lower than $0.5 \mathrm{bpp}$ )

\begin{tabular}{lcccc}
\hline Image & Sachnev et al. & Lee et al. & Dragoi and Coltuc & Proposed \\
\hline Sailboat & 42.06 & 43.59 & 43.32 & 44.10 \\
Barbara & 45.32 & 46.75 & 46.19 & 47.01 \\
Baboon & 37.60 & 38.48 & 38.32 & 38.69 \\
Boat & 42.45 & 43.92 & 44.28 & 44.64 \\
Pepper & 42.09 & 44.19 & 44.30 & 44.40 \\
Lena & 46.96 & 46.94 & 47.32 & 47.33 \\
Goldhill & 44.26 & 44.06 & 44.76 & 44.76 \\
Couple & 44.34 & 44.55 & 45.02 & 44.84 \\
House & 50.21 & 50.44 & 49.67 & 50.69 \\
Airplane & 50.80 & 49.91 & 49.89 & 50.78 \\
Elanie & 40.75 & 42.76 & 42.35 & 42.95 \\
Cameraman & 55.11 & 54.55 & 55.24 & 54.60 \\
Pirate & 39.57 & 39.77 & 41.38 & 39.94 \\
Tiffany & 47.80 & 48.30 & 47.84 & 48.33 \\
Average & 44.95 & 45.59 & 45.71 & 45.93 \\
Average gain & 0.982 & 0.344 & 0.226 & - \\
\hline
\end{tabular}

\subsubsection{Encoder}

Original image is divided into the cross set and the dot set for two-stage embedding as shown in Fig. 7. Embedding procedure starts at the cross set, and pixels are processed in raster-scanning order starting from the upper left corner. The cross set embedding procedure is as follows:

1. Compute local variance value for all pixels. Find the threshold value of the local variance values which is able to meet the embedding capacity.

2. Determine which pixels have smaller value of local variance comparing with the threshold value of local

Table 3 Comparison in terms of average PSNR(dB) for high embedding capacities(higher than $0.5 \mathrm{bpp}$ )

\begin{tabular}{lllll}
\hline Image & Sachnev et al. & Lee et al. & Dragoi and Coltuc & Proposed \\
\hline Sailboat & 33.83 & 34.38 & 34.72 & 35.13 \\
Barbara & 34.64 & 38.56 & 37.87 & 39.14 \\
Baboon & 28.38 & 29.71 & 29.17 & 29.92 \\
Boat & 34.46 & 36.37 & 36.23 & 36.92 \\
Pepper & 34.17 & 36.56 & 35.69 & 36.88 \\
Lena & 39.32 & 39.60 & 39.69 & 40.02 \\
Goldhill & 36.16 & 36.21 & 36.57 & 36.73 \\
Couple & 36.08 & 36.82 & 37.08 & 37.04 \\
House & 39.15 & 40.27 & 40.53 & 40.57 \\
Airplane & 42.25 & 42.18 & 42.17 & 42.41 \\
Elanie & 32.26 & 34.72 & 34.10 & 35.02 \\
Cameraman & 48.29 & 49.03 & 48.98 & 49.37 \\
Pirate & 32.47 & 33.02 & 33.47 & 33.20 \\
Tiffany & 38.58 & 40.42 & 39.42 & 40.45 \\
Average & 36.43 & 37.70 & 37.55 & 38.06 \\
Average gain & 1.625 & 0.354 & 0.508 & - \\
\hline
\end{tabular}



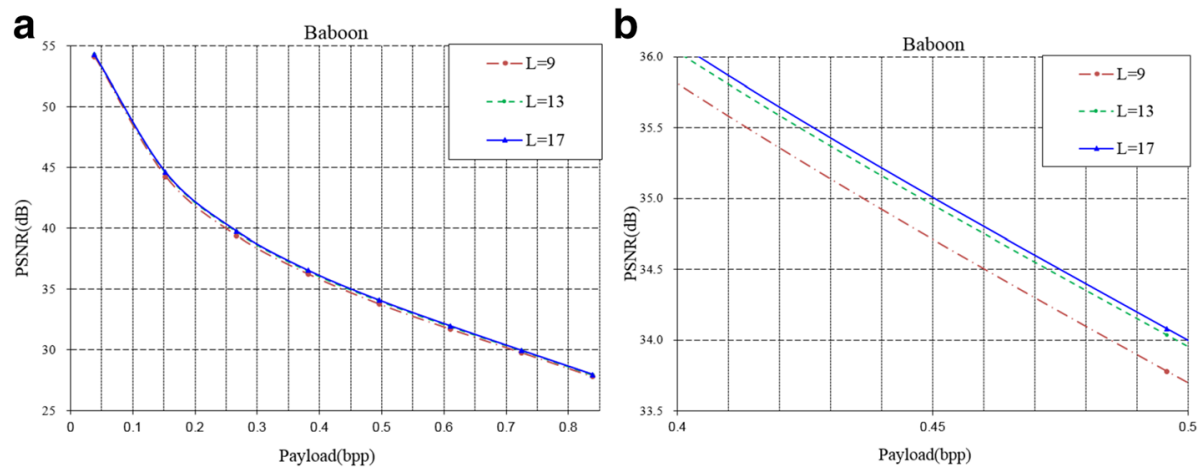

Fig. 9 PSNR vs. bpp according to training set size. a full scale, b zoomed (having 27 support pixels)

variance. Only these pixels are available for embedding.

3. Compute $x_{p}(n)$ using only a rhombus predictor [8] for the border pixels since training is not possible along the border. Compute $x_{p}(n)$ using the proposed algorithm for other pixels.

(a) Decide the training set with size $L$ centered on $y(n)$ as shown in Fig. 4.

(b) Create $X$ and $Y$ from the pixel values of the training set.

(c) Run LASSO estimator and obtain prediction coefficient $\beta$ for each support pixel.

(d)Compute $x_{p}(n)$ using Eq. (1).

4. Compute the prediction error such as $e(n)=$ $x(n)-x_{p}(n)$.

5. Embed a bit into the prediction error value using the prediction error expansion and histogram shift method.

6. Overflow and underflow problem has to be considered by using the location map bits such as Sachnev et al's method [8].

7. The pixels of the cross set are modified by embedding associated bits as shown above. The dot set embedding procedure starts with the same process. Obviously, training set includes the modified pixels of the cross set.

\subsubsection{Decoder}

Watermarked image is divided into the cross set and the dot set. Decoding procedure proceeds in the inverse order of the embedding procedure. In other words, dot set decoding proceeds first and cross set second.

The dot set decoding procedure is as follows. Obviously cross set and dot set are all modified by embedding.

1. Obtain the threshold value of the local variance, embedding capacity, and so on, from the side information.

2. Determine which pixels have smaller value of the local variance than the threshold value. Those pixels have the embedded bits.

3. In case of those pixels, compute $x_{p}(n)$ using a rhombus predictor [8] for the border pixels. Compute $x_{p}(n)$ using the proposed algorithm for other pixels.
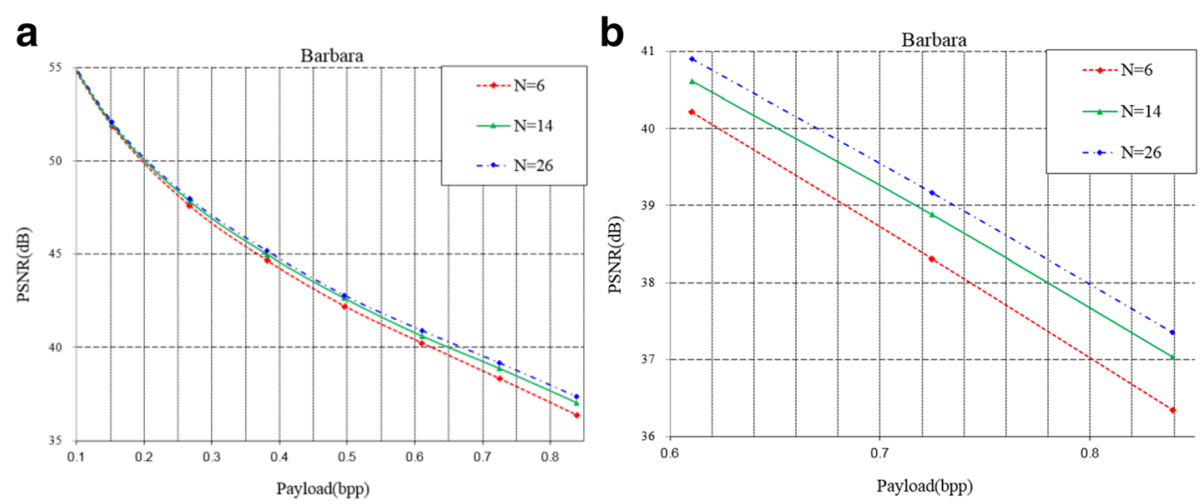

Fig. 10 PSNR vs. bpp according to the number of support pixels a full scale, $\mathbf{b}$ zoomed $(L=13)$ 

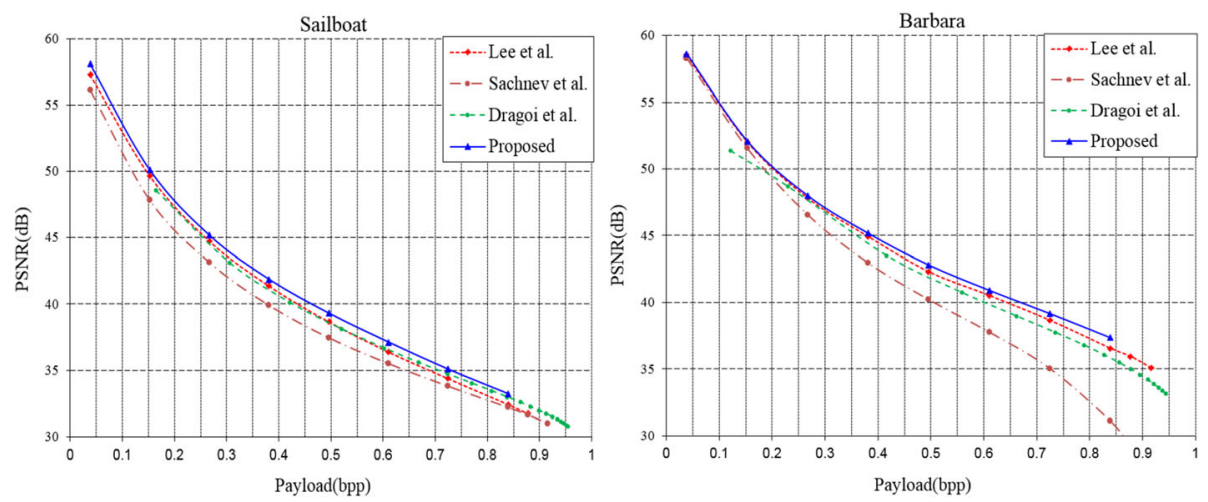

Fig. 11 PSNR comparison of other methods with the proposed method on Sailboat and Barbara

4. Compute the modified prediction error such as $e(n)=x(n)-x_{p}(n)$.

5. Extract a bit out of the modified prediction error value using the prediction error expansion and histogram shift method. Original value of the target pixel is recovered.

6. Overflow and underflow problem has to be considered by using the location map bits such as Sachnev et al's method [8].

\section{Experimental results}

We implemented the reversible data hiding algorithms of Dragoi and Dinu [9], Sachnev et al. [8], Lee at el. [20], and the proposed method using MATLAB. We tested above four algorithms implemented on well-known $512 \times 512$ sized 8-bit grayscale images such as those shown in Fig. 8; Sailboat, Barbara, Baboon, Boat, Pepper, Lena, Goldhill, Couple, and House. In addition, Airplane, Elanie, Cameraman, Pirate, and Tiffany are also utilized for further experiments in Tables 2 and 3.

We embed the watermark message and side information as binary data in the images as a payload.

\subsection{Effect of training set size, $L$}

The effect of the training set size $L$ for the ttLASSO prediction vs. PSNR performance is analyzed. In the case of Baboon, $L=17$ produces the best result as shown in Fig. 9. Without zooming, the PSNR results are almost close to each other, while significant gap exists in the zoomed result. The average PSNR difference between $L=17$ and $L=13$ is $0.038 \mathrm{~dB}$, and the average difference between 17 and 9 is $0.257 \mathrm{~dB}$.

In all above test images, the value 13 or 17 is a proper compromise as the training set size for the best results. It means that LASSO-based LS method needs to have enough training set size to obtain the best effect.

\subsection{Effect of the number of support pixel, $N$}

The effect of the number of support pixel, $N$ is quite significant. The greater number of support pixel, the better result in LASSO-based LS method. In the case of Barbara, PSNR result according to $N$ is shown in Fig. 10. The average PSNR difference between $N=26$ and $N=6$ is $0.565 \mathrm{~dB}$, and the average difference
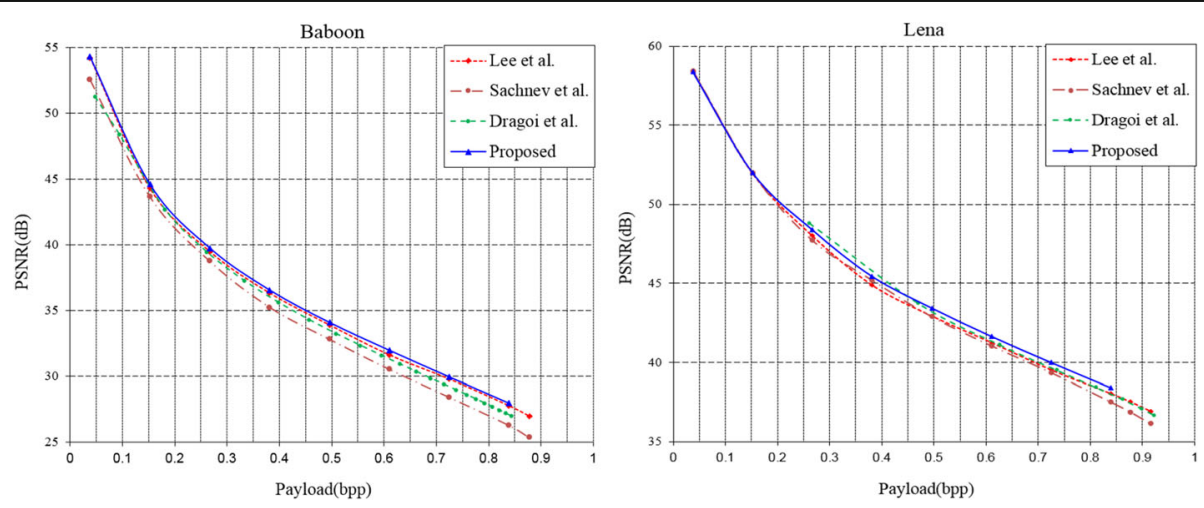

Fig. 12 PSNR comparison of other methods with the proposed method on Baboon and Lena 

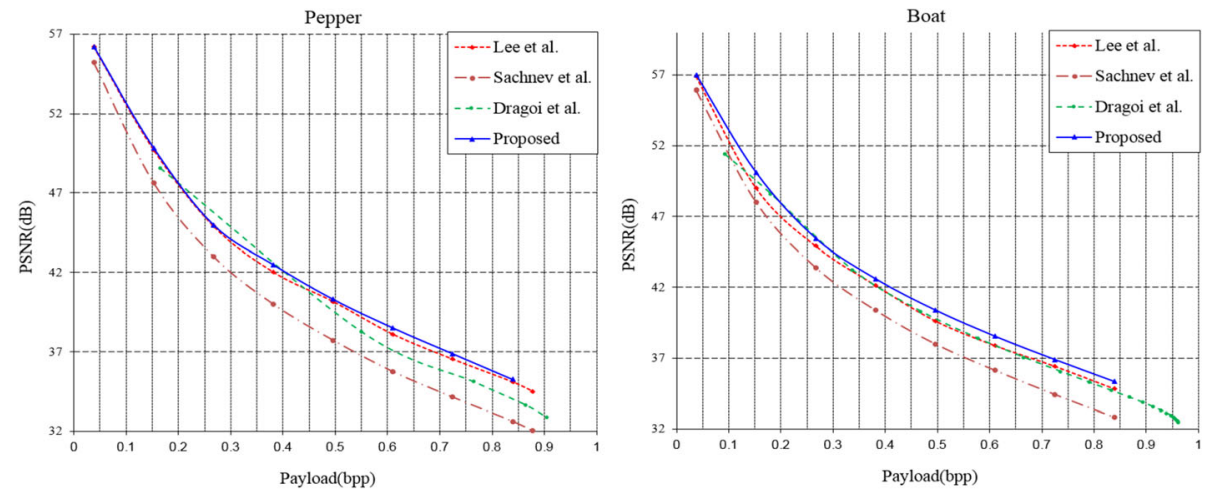

Fig. 13 PSNR comparison of other methods with the proposed method Pepper and Boat

between 26 and 14 is $0.199 \mathrm{~dB}$. In all above test images, $N=26$ has the best PSNR result. It means that LASSO-based LS method needs to have enough support pixels to obtain the best effect.

\subsection{Comparison with other state-of-the-art schemes}

Let us compare the performance of the proposed scheme with other state-of-the-art schemes such as twostage embedding scheme of [8] and other kinds of LS approaches of [9] and [20]. In the proposed method, following features are exploited:

1. LS predictor via LASSO with well-compromised size of $L$ and $N$

2. Two-stage embedding scheme with histogram shifting method [8]

The comparison results with other schemes are shown in Figs. 11, 12, 13, and 14. It is manifested that the proposed method outperforms Sachnev et al.'s method [8]. In addition, the proposed method produces slightly higher PSNR value than other least square methods [9, 20] only except a few cases of capacities on two of the test images such as Lena and Pepper. However, in terms of average gain in PSNR, the proposed method outperforms the others on all test images of Fig. 8 with $1.82 \mathrm{~dB}$ over [8], $0.59 \mathrm{~dB}$ over [9], and $0.38 \mathrm{~dB}$ over [20].

To further verify the superiority of the proposed method, experimental results for high and low embedding capacities are listed in Tables 2 and 3. The average PSNR for low embedding capacities are computed by using $40,000,70,000,100,000$, and 130,000 bits which are lower than 0.5 bpp in Table 2. On all test images of Table 2, the proposed method outperforms the others with an average gain in PSNR of $0.982 \mathrm{~dB}$ over [8], 0.344 $\mathrm{dB}$ over [20], and $0.226 \mathrm{~dB}$ over [9].

The average PSNR for high embedding capacities are computed by using 160,000, 190,000, and 220,000 bits which are higher than $0.5 \mathrm{bpp}$ in Table 3 . The result of high embedding capacities makes the superiority of the proposed method clearer. The proposed method outperforms the others with an average gain in PSNR of
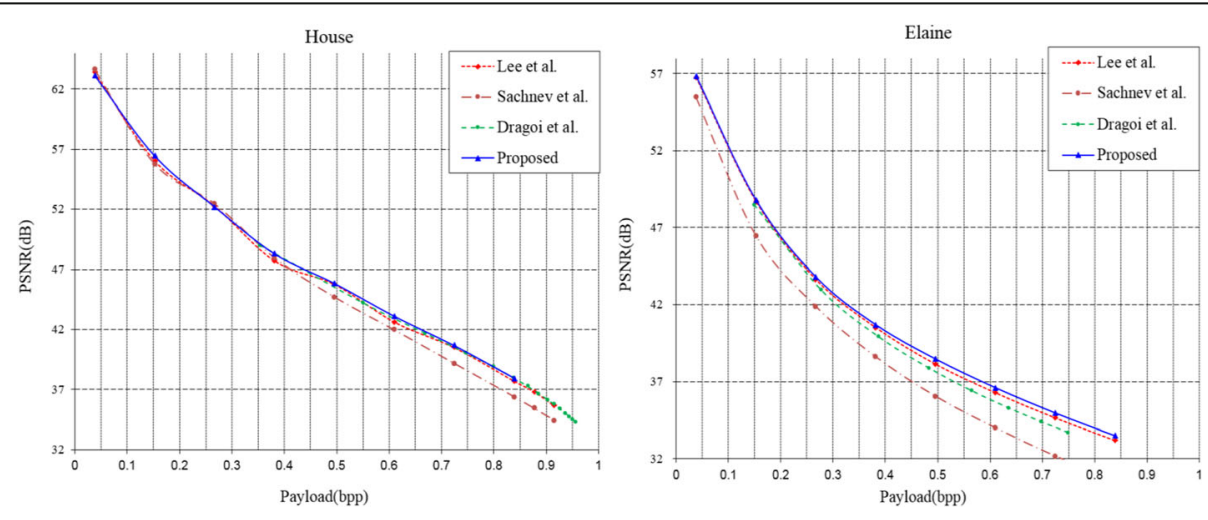

Fig. 14 PSNR comparison of other methods with the proposed method on House and Elaine 

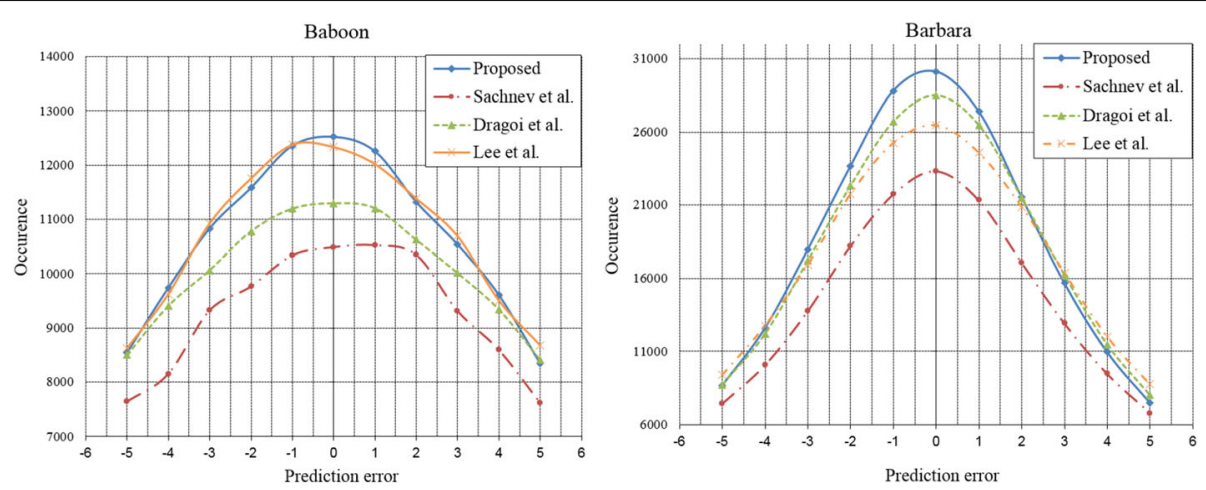

Fig. 15 Prediction error histogram comparison of other methods with the proposed method on Baboon and Barbara

$1.625 \mathrm{~dB}$ over [8], $0.354 \mathrm{~dB}$ over [20], and $0.508 \mathrm{~dB}$ over [9].

In addition, Figs. 15 and 16 confirms that the predictor in the proposed method has higher accuracy than those of Dragoi and Dinu's method and Lee et al.'s method because the proposed method results in higher occurrence of small prediction error values compared to others. The reasons why the proposed method outperforms other methods are summarized as follows.

First, the proposed method improves the state-of-theart LS predictors [9] [20] via LASSO optimization. Dragoi and Dinu's method [9] and Lee et al.'s method [20] utilize the LS predictor using the different shape of training set and support pixels. However, the proposed method applies LASSO optimization to improve the previous LS predictors. In most cases of images, the number of support pixel, $N=26$ is selected for the best prediction performance in the proposed method while $N=4$ [9] and $N=6$ [20] are used in other LS predictor. In the proposed method, LASSO optimization selects the optimized support pixels to use and remove others. In other words, the proposed method is able to utilize more proper support pixels out of many candidate support pixels to increase accuracy of the LS computation.

\section{Conclusions}

In this paper, we proposed an enhanced predictor by using LASSO approach over normal LS predictor with rhombus-shaped two-stage embedding scheme. It enables finding out the shape of region around the target pixel and the proper weight coefficients. In other words, in the proposed method, it is possible to find reasonable number and location of the support pixels due to applying LASSO into the LS approach. That is why a set of pixels located in highly variative region of image is predicted more effectively by the proposed scheme rather than other LS predictors. Due to this property, the number of high prediction errors decreases. Thus, the proposed method has a tendency that significant improvement happens in high embedding capacity, especially in highly variative images. Experimental results demonstrate that the proposed method has better results than other state-of-the-art methods.
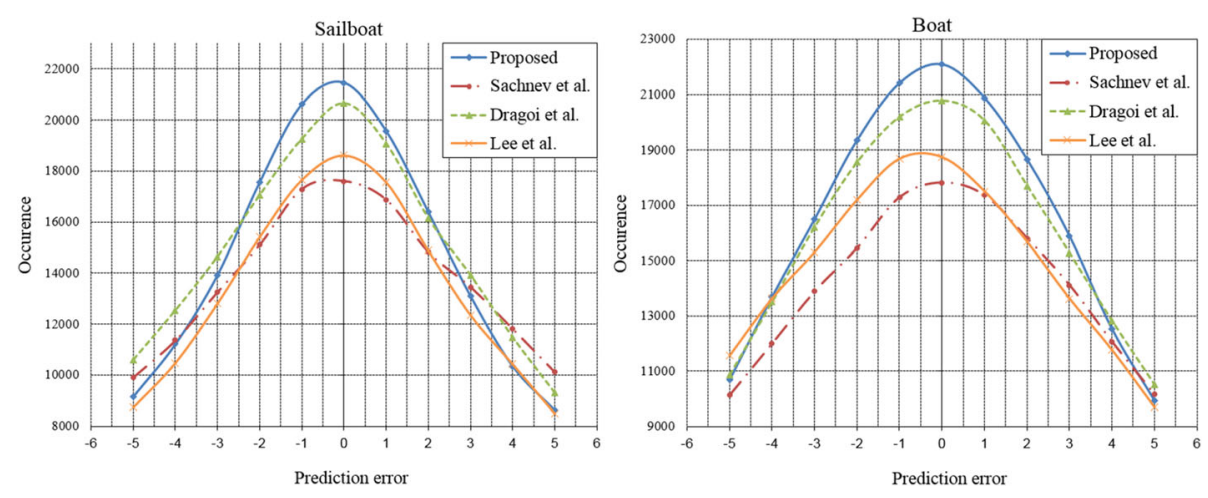

Fig. 16 Prediction error histogram comparison of other methods with the proposed method on Sailboat and Boat 


\section{Acknowledgements}

This work was supported by the National Research Foundation of Korea (NRF) grant funded by the Korea government (MEST) (NRF-2015R1A2A2A0104587).

\section{Authors' contributions}

$\mathrm{HH}$ invented the proposed idea by combining previous statistical theory with reversible watermarking prediction scheme, drafted the manuscript, and performed the statistical analysis. SK participated in the statistical theory to back up and support the proposed idea. HK participated in the design and coordination of paper and helped draft and finish manuscript of the paper.

\section{Competing interests}

The authors declare that they have no competing interests.

\section{About the authors}

Hee Joon Hwang

He received a B.S. degree in Electric and Electronic Engineering department in 2008 and a M.S. degree in Graduate School of Information Management and Security from Korea University, Seoul, Korea, in 2010. He joined at Graduate School of Information Security, Korea University, Seoul, Korea, in 2010, where he is currently pursuing Ph.D. His research interests include multimedia security, reversible and robust watermarking, and steganography. SungHwan Kim

He received a B.S. degree in Education department in 2007 and a M.S. degree in Statistics from Korea University, Seoul, Korea, in 2010. He received Ph. D. Biostatistics from University of Pittsburgh, Pittsburgh, in 2015. His research interests include methodological development for statistical machine learning methods, image processing, and optimization. Hyoung Joong Kim

He is currently with the Graduate School of Information Security, Korea University, Korea. He received his B.S., M.S., and Ph.D. from Seoul National University, Korea, in 1978, 1986, and 1989, respectively. He was a professor at Kangwon National University, Korea, from 1989 to 2006. He was a visiting scholar at University of Southern California, Los Angeles, USA, from 1992 to 1993. His research interests include data hiding such as reversible watermarking and steganography.

\section{Author details}

'Graduate School of Information Security, Korea University, Seoul, South Korea. ${ }^{2}$ Department of Statistics, Korea University, Seoul, South Korea.

Received: 1 June 2016 Accepted: 10 November 2016

Published online: 07 December 2016

\section{References}

1. J Tian, Reversible data embedding using a difference expansion. IEEE Trans. Circuits Syst. Video Technol. 13(8), 890-896 (2003)

2. AM Alattar, Reversible watermark using difference expansion of triplets, in Proc. IEEE Int. Conf. Image Process. IEEE International Conference on Image Processing, Catalonia, Spain, 2003, vol. 1, pp. 501-504

3. AM Alattar, Reversible watermark using the difference expansion of a generalized integer transform. IEEE Trans. Image Process. 13, 1147-1156 (2004)

4. DM Thodi, JJ Rodriguez, Expansion embedding techniques for reversible watermarking. IEEE Trans. Image Process 16(3), 721-730 (2007)

5. MJ Weinberger, G Seroussi, G Sapiro, The LOCO-I lossless image compression algorithm:principles and standardization into JPEG-LS. IEEE Trans. Image Process. 9(8), 1309-1324 (2000)

6. $\mathrm{M}$ Chen, Z Chen, $X$ Zeng, Z Xiong, Model order selection in reversible image watermarking. IEEE J. Sel. Top. Signal Process. 4(3), 592-604 (2010)

7. X Wu, N Memon, Context-based, adaptive, lossless image coding. IEEE Trans. Commun. 45(4), 437-444 (1997)

8. V Sachnev, HJ Kim, J Nam, S Suresh, YQ Shi, Reversible watermarking algorithm using sorting and prediction. IEEE Trans. Circuits Syst. Video Technol. 19(7), 989-999 (2009)

9. IC Dragoi, D Coltuc, Local-prediction-based difference expansion reversible watermarking. IEEE Trans. Image Process. 23(4), 1779-1790 (2014)

10. HJ Hwang, HJ Kim, V Sachnev, SH Joo, Reversible watermarking method using optimal histogram pair shifting based on prediction and sorting. KSII, Trans. Internet Inform. Syst. 4(4), 655-670 (2010)

11. SU Kang, HJ Hwang, HJ Kim, Reversible watermark using an accurate predictor and sorter based on payload balancing. ETRI J. 34(3), 410-420 (2012)
12. G Feng, Z Qian, N Dai, Reversible watermarking via extreme learning machine prediction. Neurocomputing 82(1), 62-68 (2012)

13. L Luo, Z Chen, M Chen, X Zeng, Z Xiong, Reversible image watermarking using interpolation technique. IEEE Trans. Inf. Forensics Secur 5(1), 187-193 (2010). 5010-5021

14. B Ou, X Li, Y Zhao, R Ni, YQ Shi, Pairwise prediction-error expansion for efficient reversible data hiding. IEEE Trans. Image Process. 22(12), 36-42 (2013)

15. S. Weng, and J.S. Pan, Reversible watermarking based on two embedding schemes, Multimedia Tools Appl. 2016;75(12):7129-157.

16. IC Dragoi, D Coltuc, Adaptive pairing reversible watermarking. IEEE Trans. Image Process. 25(5), 2420-2422 (2016)

17. L-J Kau, Y-P Lin, Adaptive lossless image coding using least squares optimization with edge-look-ahead. IEEE Trans. Circuits Syst. 52(11), 751-755 (2005)

18. X Wu, G Zhai, X Yang, W Zhang, Adaptive sequential prediction of multidimensional signals with applications to lossless image coding. IEEE Trans. Image Process. 20(1), 36-42 (2011)

19. J. Wen, L. Jinli, and W. Yi Adaptive reversible data hiding through autoregression, In Proceedings of 2012 IEEE International Conference on Information Science and Technology, ICIST. 2012. pp.831-838.

20. BY Lee, HJ Hwang, HJ Kim, Reversible data hiding using piecewise autoregresive predictor based on two-stage embedding. J. Elect. Eng. Tech. 11(4), 974-986 (2016)

21. X Li, MT Orchard, Edge-directed prediction for lossless compression of natural images. IEEE Trans. Image Process. 10(6), 813-817 (2001)

22. Z Ni, Y-Q Shi, N Ansari, W Su, Reversible data hiding. IEEE Trans, Circuits Syst. 16(3), 354-365 (2006)

23. Y-Q Shi, X Li, X Zhang, H-T Wu, B Ma, Reversible data hiding: advances in the past two decades. IEEE Access 4, 3210-3237 (2016)

24. R Tibshirani, Regression shrinkage and selection via the lasso. J. Royal Stat. Soc., Series B 58(1), 267-288 (1996)

25. G Schwarz, Estimating the dimension of a model. Ann Stat 6(2), 461-464 (1978)

\section{Submit your manuscript to a SpringerOpen ${ }^{\circ}$ journal and benefit from:}

- Convenient online submission

- Rigorous peer review

- Immediate publication on acceptance

- Open access: articles freely available online

- High visibility within the field

- Retaining the copyright to your article

Submit your next manuscript at $>$ springeropen.com 